\title{
Inventory Model: Deteriorating Items with Time-Dependent Deterioration Rate for Quadratic Demand Rate With Unit Production Cost and Shortage
}

\author{
Jasvinder Kaur ${ }^{1}$, A.P.Singh ${ }^{2}$, Rajendra Sharma ${ }^{3}$
}

\begin{abstract}
In this paper, an order level inventory unit production system for deteriorating items with a quadratic demand is developed. It is assumed that shortages in inventory are permitted and are completely backlogged. The finite production rate is proportional to the time dependent demand rate and deterioration rate is time proportional. The unit production cost is taken to be inversely related to the demand rate. Sensitivity of the decision variable to changes in the parameter values is examined and numerical example is presented to illustrate the model is developed.
\end{abstract}

Keywords - Deterioration; Inventory model; Quadratic demand rate; Shortage;

\section{INTRODUCTION}

In general, deterioration is defined as the damage, spoilage, dryness, vaporization, etc., that result in decrease of usefulness of the original one. Goods deteriorate and their value reduces with time. Electronic products may become absolute as technology changes. Fashion tends to depreciate the value of clothing over time .Batteries dies out as they age. The effect of time is even more critical for perishable goods such as foods stuffs and cigarettes

Whitin (1957) considered fashion goods deteriorating at the end of a prescribed shortage period. Ghare and Schrader (1963) develop a model for an exponentially decaying inventory. Various types of order-level inventory models for items deteriorating at a constant were discussed by Shah and Jaiswal (1977), Dave and Patel (1981), Roychowdhury and Chaudhuri (1983), Dave (1986) and Bahari-kashani (1989). Inventory models with a time-dependent rate of deterioration were studied by Covert and Philip (1973), Philip (1974), Mishra (1975) and Deb and Chaudhuri (1986).

Numerous inventory models for deteriorating items with various features of the inventory systems have been discussed; it is not possible to list them all. Interested readers may consult the review articles by Nahmias (1982) and Raafat (1991). In the classical inventory models, the demand rate is assumed to be a constant. In reality, demand for physical goods may be time dependent, stock dependent and price dependent. The first analytic model for linearly time-dependent demand was developed by Donaldson (1977). Most of these papers take the replenishment rate to be infinite.

In this present paper, we discuss an economic order quantity (EOQ) model taking into account the following factors

1. The production rate is finite and proportional to the time dependent quadratic demand rate

2. The unit cost of production depends on the demand rate.

3. The deterioration rate is time proportional.

\section{Assumptions}

\section{Proposed ASSUMtions \& Notations}

A deterministic order-level model with a finite rate of replenishment is developed with the following assumptions and notations.

$1.1 c_{1}$ is the constant holding cost per item per unit of time.

1.2 The shortage cost $c_{2}$ is infinite.

$1.3 c_{3}$ is the constant deterioration cost per unit per unit of time.

$1.4 R=f(t)=a+b t+c t^{2}$ is the quadratic demand rate at any time $t \geq 0, a \geq 0, b>0, c>0$.

$1.5 K=\beta f(t)$ is the production rate where $\beta(>1)$ is a constant.

1.6 The lead time is zero.

1.7 A variable fraction $\theta(t)=\alpha t,(0<\alpha<<1, t \geq 0)$ of the on-hand inventory deteriorates per unit of time.

$1.8 \mathrm{C}$ is the total average cost for a production cycle. 
1.9 The unit production cost $v$ is inversely related to the demand rate as $v=\alpha_{1} R^{-\gamma}$, where $\alpha_{1}>0, \gamma>0$ and $\gamma \neq 2 . \quad \alpha_{1}$ is obviously positive since $v$ and $R$ are both non-negative; also higher demands result in lower unit costs of production. This implies that $v$ and $R$ are inversely related and, hence, $\gamma$ must be positive.

Now, $\quad \frac{d v}{d R}=-\alpha_{1} \gamma R^{-(\gamma+1)}<0$,

$$
\frac{d^{2} v}{d R^{2}}=\alpha_{1} \gamma(\gamma+1) R^{-(\gamma+2)}>0 \text {. }
$$

Thus marginal unit cost of production is an increasing function of $R$. These results imply that, as the demand rate increases, the unit cost of production decreases at an increasing rate. For this reason, the manufacturer is encouraged to produce more as the demand for the item increases. The necessity of the restriction $\gamma \neq 2$ arises from the nature of the solution of problem. The amount of stock is zero at time $t=0$. Production starts at time $t=0$ and stops at time $t_{1}$ when the stock attains a level $S$. During $\left[t_{1}, t_{2}\right]$, the inventory level gradually decreases mainly to meet demands and partly because of deterioration. By this process, the stock reaches zero level at time $t_{2}$. The cycle then repeats itself after the scheduling period $t_{2}$. The intensity of deterioration is very low during the early stage of inventory because $t$ is small. However, the intensity increases with time, but $\theta(t)$ remains bounded for $t>>1$ since $0<\alpha<<1$.

In this case, we intend to develop an order-level model for deteriorating items with a finite rate of replenishment with the assumptions described and also with the additional assumption that shortages in inventory are permitted and are completely backlogged. $c_{2}$ is the constant shortage per unit per unit of time.

Here the amount of stock is zero at time $t=0$. Production starts at time $t=0$ and continues up to $t=t_{1}$ when the stock reaches a level $S$. Inventory accumulated in $\left[0, t_{1}\right]$ after meeting the quadratic demands is used in $\left[t_{1}, t_{2}\right]$. The stock reaches the zero level at time $t_{2}$. Now shortages start to develop and accumulate to the level $P$ at $t=t_{3}$. Production starts at time $t_{3}$. The running demands as well as the backlog for $\left[t_{2}, t_{3}\right]$ are satisfied in $\left[t_{3}, t_{4}\right]$. The inventory again falls to the zero level at time $t_{4}$. The cycle then repeats itself after a time $t_{4}$. Our problem is to determine the optimum values of $t_{1}, t_{2}, t_{3}, t_{4}$ and $C$ with the assumptions stated above.

\section{MATHEMATICAL FORMULATION:}

\section{INDENTATIONS AND EQUATIONS}

Let $Q(t)$ represent the instantaneous inventory level at any time $t\left(0 \leq t \leq t_{4}\right)$. The differential equations governing the instantaneous states of $Q(t)$ in the interval $\left[0, t_{4}\right]$ are given by

$$
\frac{d Q(t)}{d t}+\theta(t) Q(t)=K-f(t), \quad 0 \leq t \leq t_{1},
$$

with the conditions $Q(0)=0$ and $Q\left(t_{1}\right)=S$;

$$
\frac{d Q(t)}{d t}+\theta(t) Q(t)=-f(t), \quad t_{1} \leq t \leq t_{2}
$$

with the conditions $Q\left(t_{1}\right)=S$ and $Q\left(t_{2}\right)=0$.

$$
\frac{d Q(t)}{d t}=-f(t), \quad t_{2} \leq t \leq t_{3},
$$

with the conditions $Q\left(t_{2}\right)=0$ and $Q\left(t_{3}\right)=-P$; 


$$
\frac{d Q(t)}{d t}=K-f(t), \quad t_{3} \leq t \leq t_{4} .
$$

with the conditions $Q\left(t_{3}\right)=-P$ and $Q\left(t_{4}\right)=0$.

Using $\theta(t)=\alpha t$ and $f(t)=a+b t+c t^{2}$, equation (1)-(4) become respectively

$$
\frac{d Q(t)}{d t}+\alpha t Q(t)=(\beta-1)\left(a+b t+c t^{2}\right), \quad 0 \leq t \leq t_{1},
$$

with the conditions $Q(0)=0$ and $Q\left(t_{1}\right)=S$;

$$
\frac{d Q(t)}{d t}+\alpha t Q(t)=-\left(a+b t+c t^{2}\right), \quad t_{1} \leq t \leq t_{2} ，
$$

with the conditions $Q\left(t_{1}\right)=S$ and $Q\left(t_{2}\right)=0$;

$$
\frac{d Q(t)}{d t}=-\left(a+b t+c t^{2}\right), \quad t_{2} \leq t \leq t_{3},
$$

with the conditions $Q\left(t_{2}\right)=0$ and $Q\left(t_{3}\right)=-P$;

$$
\frac{d Q(t)}{d t}=(\beta-1)\left(a+b t+c t^{2}\right), \quad t_{3} \leq t \leq t_{4} .
$$

with the conditions $Q\left(t_{3}\right)=-P$ and $Q\left(t_{4}\right)=0$.

The solutions of (5)-(8) are respectively.

$$
\begin{aligned}
& Q(t)=(\beta-1)\left(a t-\frac{1}{3} a \alpha t^{3}+\frac{1}{2} b t^{2}-\frac{1}{8} b \alpha t^{4}+\frac{1}{3} c t^{3}-\frac{1}{15} c \alpha t^{5}\right), \quad 0 \leq t \leq t_{1}, \\
& Q(t)=\left\{\begin{array}{c}
S\left(1+\frac{1}{2} \alpha t_{1}^{2}-\frac{1}{2} \alpha t^{2}\right)+a\left(t_{1}-t+\frac{1}{6} \alpha t_{1}^{3}-\frac{1}{2} \alpha t^{2} t_{1}+\frac{1}{3} \alpha t^{3}\right) \\
\quad+b\left(\frac{1}{2} t_{1}^{2}-\frac{1}{2} t^{2}+\frac{1}{8} \alpha t_{1}^{4}-\frac{1}{4} \alpha t^{2} t_{1}^{2}+\frac{1}{8} \alpha t^{4}\right) \\
\quad+c\left(\frac{1}{3} t_{1}^{3}-\frac{1}{3} t^{3}+\frac{1}{10} \alpha t_{1}^{5}-\frac{1}{6} \alpha t^{2} t_{1}^{3}+\frac{1}{15} \alpha t^{5}\right), \quad t_{1} \leq t \leq t_{2} .
\end{array}\right. \\
& Q(t)=a\left(t_{2}-t\right)+\frac{1}{2} b\left(t_{2}^{2}-t^{2}\right)+\frac{1}{3} c\left(t_{2}^{3}-t^{3}\right), t_{2} \leq t \leq t_{3}, \\
& Q(t)=(\beta-1)\left[a\left(t-t_{4}\right)+\frac{1}{2} b\left(t^{2}-t_{4}^{2}\right)+\frac{1}{3} c\left(t^{3}-t_{4}^{3}\right)\right], \quad t_{3} \leq t \leq t_{4} .
\end{aligned}
$$

Since $Q\left(t_{2}\right)=0$, then from (10),

$$
\begin{aligned}
S=a\left(t_{2}-t_{1}+\frac{1}{3} \alpha t_{1}^{3}+\frac{1}{6} \alpha t_{2}^{3}-\frac{1}{2} \alpha t_{1}{ }^{2} t_{2}\right) \\
+b\left(\frac{1}{2} t_{2}{ }^{2}-\frac{1}{2} t_{1}{ }^{2}+\frac{1}{8} \alpha t_{1}^{4}+\frac{1}{8} \alpha t_{2}^{4}-\frac{1}{4} \alpha t_{1}{ }^{2} t_{2}{ }^{2}\right) \\
+c\left(\frac{1}{3} t_{2}{ }^{3}-\frac{1}{3} t_{1}{ }^{3}+\frac{1}{15} \alpha t_{1}^{5}+\frac{1}{10} \alpha t_{2}{ }^{5}-\frac{1}{6} \alpha t_{1}{ }^{2} t_{2}{ }^{3}\right) .
\end{aligned}
$$

for a first-order approximation of $\alpha$.

The total inventory in the cycle $\left[0, t_{2}\right]$ is 


$$
\begin{aligned}
\int_{0}^{t_{1}} Q(t) d t+\int_{t_{1}}^{t_{2}} Q(t) d t & =\frac{1}{12} a\left[6 \beta t_{1}^{2}-\alpha \beta t_{1}^{4}+6 t_{2}{ }^{2}-12 t_{1} t_{2}+2 \alpha t_{1}{ }^{3} t_{2}+\alpha t_{2}{ }^{4}-2 \alpha t_{1} t_{2}{ }^{3}\right] \\
+ & \frac{1}{120} b\left[20 \beta t_{1}{ }^{3}-3 \alpha \beta t_{1}{ }^{5}+40 t_{2}{ }^{3}-60 t_{1} t_{2}{ }^{2}+10 \alpha t_{1}{ }^{3} t_{2}{ }^{2}+8 \alpha t_{2}{ }^{5}-15 \alpha t_{1} t_{2}{ }^{4}\right] \\
+ & \frac{1}{180} c\left[15 \beta t_{1}{ }^{4}-2 \alpha \beta t_{1}{ }^{6}+45 t_{2}{ }^{4}-60 t_{1} t_{2}{ }^{3}+10 \alpha t_{1}{ }^{3} t_{2}{ }^{3}+10 \alpha t_{2}{ }^{6}-18 \alpha t_{1} t_{2}{ }^{5}\right]
\end{aligned}
$$

for a first-order approximation of $\alpha$.

The total number of deteriorated items in $\left[0, t_{2}\right]$ is given by

Production in $\left[0, t_{1}\right]$-demand in

$$
\begin{aligned}
{\left[0, t_{2}\right] } & =\beta \int_{0}^{t_{1}}\left(a+b t+c t^{2}\right) d t-\int_{0}^{t_{2}}\left(a+b t+c t^{2}\right) d t \\
& =a\left(\beta t_{1}-t_{2}\right)+\frac{1}{2} b\left(\beta t_{1}{ }^{2}-t_{2}{ }^{2}\right)+\frac{1}{3} c\left(\beta t_{1}{ }^{3}-t_{2}{ }^{3}\right)
\end{aligned}
$$

Since the production in $[u, u+d u]$ is $K d u$, the cost of production in $[u, u+d u]$ is

$$
K v d u=\frac{\alpha_{1} \beta f(t)}{R^{\gamma}} d u=\frac{\alpha_{1} \beta}{\left(a+b u+c u^{2}\right)^{\gamma-1}} d u
$$

Hence the production cost in $\left[0, t_{1}\right]$ is

$$
\int_{0}^{t_{1}} \frac{\alpha_{1} \beta}{\left(a+b u+c u^{2}\right)^{\gamma-1}} d u=\frac{\alpha_{1} \beta}{6 a^{\gamma}}\left[6 a t_{1}+(1-\gamma)\left(3 b t_{1}^{2}+2 c t_{1}^{3}\right)\right], \quad \gamma \neq 2 .
$$

The total number of deteriorated items in $\left[0, t_{4}\right]$ is the same as given in (13) since there will be no deteriorated items during the period $\left[t_{2}, t_{4}\right]$.

The total shortage in $\left[t_{2}, t_{4}\right]$ is given by

$$
\begin{aligned}
\int_{t_{2}}^{t_{4}}[-Q(t)] d t= & \int_{t_{2}}^{t_{3}}[-Q(t)] d t+\int_{t_{3}}^{t_{4}}[-Q(t)] d t \\
= & \frac{1}{2} a\left[t_{2}{ }^{2}+\beta t_{3}{ }^{2}+(\beta-1) t_{4}{ }^{2}-2 t_{2} t_{3}-2(\beta-1) t_{3} t_{4}\right] \\
& +\frac{1}{6} b\left[2 t_{2}{ }^{3}+\beta t_{3}{ }^{3}+2(\beta-1) t_{4}{ }^{3}-3 t_{2}{ }^{2} t_{3}-3(\beta-1) t_{3} t_{4}{ }^{2}\right] \\
& +\frac{1}{12} c\left[3 t_{2}{ }^{4}+\beta t_{3}{ }^{4}+3(\beta-1) t_{4}{ }^{4}-4 t_{2}{ }^{3} t_{3}-4(\beta-1) t_{3} t_{4}{ }^{3}\right]
\end{aligned}
$$

The production cost in $\left[t_{3}, t_{4}\right]$ is 


$$
\begin{aligned}
\int_{t_{3}}^{t_{4}} K v d u=\alpha_{1} \beta \int_{t_{3}}^{t_{4}}\left(a+b u+c u^{2}\right)^{1-\gamma} d u \\
\quad=\frac{\alpha_{1} \beta}{6 a^{\gamma}}\left[6 a\left(t_{4}-t_{3}\right)+(1-\gamma)\left(3 b\left(t_{4}{ }^{2}-t_{3}{ }^{2}\right)+2 c\left(t_{4}{ }^{3}-t_{3}{ }^{3}\right)\right)\right], \quad \gamma \neq 2 .
\end{aligned}
$$

Therefore, the production cost in $\left[0, t_{4}\right]$ is

$$
\begin{gathered}
\frac{\alpha_{1} \beta}{6 a^{\gamma}}\left[6 a t_{1}+(1-\gamma)\left(3 b t_{1}{ }^{2}+2 c t_{1}{ }^{3}\right)\right]+\frac{\alpha_{1} \beta}{6 a^{\gamma}}\left[6 a\left(t_{4}-t_{3}\right)+(1-\gamma)\left(3 b\left(t_{4}{ }^{2}-t_{3}{ }^{2}\right)+2 c\left(t_{4}{ }^{3}-t_{3}{ }^{3}\right)\right)\right] \\
\quad=\frac{\alpha_{1} \beta}{6 a^{\gamma}}\left[6 a\left(t_{1}+t_{4}-t_{3}\right)+3 b(1-\gamma)\left(t_{1}{ }^{2}+t_{4}{ }^{2}-t_{3}{ }^{2}\right)+2 c(1-\gamma)\left(t_{1}{ }^{3}+t_{4}{ }^{3}-t_{3}{ }^{3}\right)\right], \quad \gamma \neq 2 .
\end{gathered}
$$

The total average cost of the system in $\left[0, t_{4}\right]$ is

$$
\begin{aligned}
C=\frac{1}{t_{4}}[ & \frac{1}{12} a c_{1}\left[6 \beta t_{1}{ }^{2}-\alpha \beta t_{1}^{4}+6 t_{2}{ }^{2}-12 t_{1} t_{2}+2 \alpha t_{1}{ }^{3} t_{2}+\alpha t_{2}{ }^{4}-2 \alpha t_{1} t_{2}{ }^{3}\right] \\
& +\frac{1}{120} b c_{1}\left[20 \beta t_{1}{ }^{3}-3 \alpha \beta t_{1}{ }^{5}+40 t_{2}{ }^{3}-60 t_{1} t_{2}{ }^{2}+10 \alpha t_{1}{ }^{3} t_{2}{ }^{2}+8 \alpha t_{2}{ }^{5}-15 \alpha t_{1} t_{2}{ }^{4}\right] \\
& +\frac{1}{180} c c_{1}\left[15 \beta t_{1}^{4}-2 \alpha \beta t_{1}{ }^{6}+45 t_{2}{ }^{4}-60 t_{1} t_{2}{ }^{3}+10 \alpha t_{1}{ }^{3} t_{2}{ }^{3}+10 \alpha t_{2}{ }^{6}-18 \alpha t_{1} t_{2}{ }^{5}\right] \\
& +\frac{1}{2} a c_{2}\left[t_{2}{ }^{2}+\beta t_{3}{ }^{2}+(\beta-1) t_{4}{ }^{2}-2 t_{2} t_{3}-2(\beta-1) t_{3} t_{4}\right] \\
& +\frac{1}{6} b c_{2}\left[2 t_{2}{ }^{3}+\beta t_{3}{ }^{3}+2(\beta-1) t_{4}{ }^{3}-3 t_{2}{ }^{2} t_{3}-3(\beta-1) t_{3} t_{4}{ }^{2}\right] \\
& +\frac{1}{12} c c_{2}\left[3 t_{2}{ }^{4}+\beta t_{3}{ }^{4}+3(\beta-1) t_{4}{ }^{4}-4 t_{2}{ }^{3} t_{3}-4(\beta-1) t_{3} t_{4}{ }^{3}\right] \\
& +a c_{3}\left(\beta t_{1}-t_{2}\right)+\frac{1}{2} b c_{3}\left(\beta t_{1}{ }^{2}-t_{2}{ }^{2}\right)+\frac{1}{3} c c_{3}\left(\beta t_{1}{ }^{3}-t_{2}{ }^{3}\right) \\
& +\frac{\alpha_{1} \beta}{6 a^{\gamma}}\left[6 a\left(t_{1}+t_{4}-t_{3}\right)+3 b(1-\gamma)\left(t_{1}{ }^{2}+t_{4}{ }^{2}-t_{3}{ }^{2}\right)+2 c(1-\gamma)\left(t_{1}{ }^{3}+t_{4}{ }^{3}-t_{3}{ }^{3}\right)\right]
\end{aligned}
$$

The optimums values of $t_{1}, t_{2}, t_{3}$ and $t_{4}$ which minimize the cost function $C$ are the solutions of the equations

$$
\frac{\partial C}{\partial t_{1}}=0, \frac{\partial C}{\partial t_{2}}=0, \frac{\partial C}{\partial t_{3}}=0 \text { and } \frac{\partial C}{\partial t_{4}}=0
$$

provided that these values of $t_{i}(i=1,2,3,4)$ satisfy the conditions $D_{i}>0(i=1,2,3,4)$, where $D_{i}$ is the Hessian determinant of order $i$ given by

$$
D_{i}=\left|\begin{array}{cccc}
c_{11} & c_{12} & \cdots & c_{1 i} \\
c_{21} & c_{22} & \cdots & c_{2 i} \\
\vdots & \vdots & \cdots & \vdots \\
c_{i 1} & c_{i 2} & \cdots & c_{i i}
\end{array}\right|,
$$




$$
c_{i j}=\frac{\partial^{2} C}{\partial t_{i} \partial t_{j}} \quad(i, j=1,2,3,4) .
$$

The expanded forms of the (16) are

$$
\begin{aligned}
\frac{1}{12} a c_{1}[ & \left.12 \beta t_{1}-4 \alpha \beta t_{1}{ }^{3}-12 t_{2}+6 \alpha t_{1}{ }^{2} t_{2}-2 \alpha t_{2}{ }^{3}\right] \\
& +\frac{1}{120} b c_{1}\left[60 \beta t_{1}{ }^{2}-15 \alpha \beta t_{1}^{4}-60 t_{2}{ }^{2}+30 \alpha t_{1}{ }^{2} t_{2}{ }^{2}-15 \alpha t_{2}{ }^{4}\right] \\
& +\frac{1}{180} c c_{1}\left[60 \beta t_{1}{ }^{3}-12 \alpha \beta t_{1}{ }^{5}-60 t_{2}{ }^{3}+30 \alpha t_{1}{ }^{2} t_{2}{ }^{3}-18 \alpha t_{2}{ }^{5}\right] \\
& +a c_{3} \beta+b c_{3} \beta t_{1}+c c_{3} \beta t_{1}{ }^{2}+\frac{\alpha_{1} \beta}{a^{\gamma}}\left[a+(1-\gamma) b t_{1}+(1-\gamma) c t_{1}{ }^{2}\right]=0, \gamma \neq 2, \\
\frac{1}{12} a c_{1}[ & \left.12 t_{2}-12 t_{1}+2 \alpha t_{1}{ }^{3}+4 \alpha t_{2}{ }^{3}-6 \alpha t_{1} t_{2}{ }^{2}\right] \\
& +\frac{1}{120} b c_{1}\left[120 t_{2}{ }^{2}-120 t_{1} t_{2}+20 \alpha t_{1}{ }^{3} t_{2}+40 \alpha t_{2}{ }^{4}-60 \alpha t_{1} t_{2}{ }^{3}\right] \\
& +\frac{1}{180} c c_{1}\left[180 t_{2}{ }^{3}-180 t_{1} t_{2}{ }^{2}+30 \alpha t_{1}{ }^{3} t_{2}{ }^{2}+60 \alpha t_{2}{ }^{5}-90 \alpha t_{1} t_{2}{ }^{4}\right] \\
& +a c_{2}\left(t_{2}-t_{3}\right)+b c_{2}\left(t_{2}{ }^{2}-t_{2} t_{3}\right)+c c_{2}\left(t_{2}{ }^{3}-t_{2}{ }^{2} t_{3}\right)-a c_{3}-b c_{3} t_{2}-c c_{3} t_{2}{ }^{2}=0, \\
& +\frac{\alpha_{1} \beta}{a^{\gamma}}\left[a+(1-\gamma) b t_{4}+(1-\gamma) c t_{4}{ }^{2}\right]-C=0 . \\
a c_{2}\left[\beta t_{3}\right. & \left.-t_{2}-(\beta-1) t_{4}\right]+\frac{1}{2} b c_{2}\left[\beta t_{3}{ }^{2}-t_{2}{ }^{2}-(\beta-1) t_{4}{ }^{2}\right] \\
+ & \frac{1}{3} c c_{2}\left[\beta t_{3}{ }^{3}-t_{2}{ }^{3}-(\beta-1) t_{4}{ }^{3}\right]-\frac{\alpha_{1} \beta}{a^{\gamma}}\left[a+(1-\gamma) b t_{3}+(1-\gamma) c t_{3}{ }^{2}\right]=0,
\end{aligned}
$$

Example - Let $a=0.4, b=0.23, c=0.05, c_{1}=40, c_{2}=120, c_{3}=150, \alpha=0.02, \beta=8, \alpha_{1}=50$ and $\gamma$ $=1.5$ in appropriate units. By applying Mathematica 4.1, we obtain the optimum solutions for $t_{1}, t_{2}, t_{3}$ and $t_{4}$ of equations (17)-(20) as $t_{1}{ }^{*}=12.79, t_{2}{ }^{*}=18.62, t_{3}{ }^{*}=23.51$ and $t_{4}{ }^{*}=25.32$. Substituting $t_{1}{ }^{*}, t_{2}{ }^{*}, t_{3}{ }^{*}$ and $t_{4}{ }^{*}$ in equation (15), we obtain the optimum average cost as $C^{*}=34,026.70$. 
Inventory Model: Deteriorating Items with Time-Dependent Deterioration Rate for Quadratic

Table 1

\begin{tabular}{|c|c|c|c|c|c|c|}
\hline $\begin{array}{l}\text { Changing } \\
\text { Parameter }\end{array}$ & (\%) change & $t_{1}^{*}$ & $t_{2}{ }^{*}$ & $t_{3}{ }^{*}$ & $t_{4}{ }^{*}$ & $C^{*}$ \\
\hline \multirow{4}{*}{$\alpha$} & +50 & 12.63 & 18.70 & 22.17 & 23.15 & $35,207.20$ \\
\hline & +25 & 12.86 & 18.58 & 22.77 & 24.13 & $34,385.20$ \\
\hline & -25 & 12.95 & 18.49 & 25.14 & 27.19 & $33,384.13$ \\
\hline & -50 & 13.54 & 18.35 & 26.31 & 31.52 & $31,148.60$ \\
\hline \multirow{4}{*}{$\beta$} & +50 & 13.36 & 19.37 & 25.56 & 27.45 & $41,639.60$ \\
\hline & +25 & 13.21 & 19.02 & 24.62 & 26.41 & $39,528.40$ \\
\hline & -25 & 12.65 & 17.79 & 22.62 & 24.17 & $34,030.20$ \\
\hline & -50 & 12.13 & 17.26 & 21.54 & 22.35 & $31,481.40$ \\
\hline \multirow{4}{*}{$\alpha_{1}$} & +50 & 12.54 & 17.66 & 24.14 & 26.51 & $44,629.60$ \\
\hline & +25 & 12.74 & 18.07 & 23.83 & 25.71 & $39,241.70$ \\
\hline & -25 & 13.16 & 19.52 & 23.25 & 25.15 & $28,378.10$ \\
\hline & -50 & 13.67 & 21.09 & 22.82 & 24.62 & $22,312.50$ \\
\hline \multirow{4}{*}{$\gamma$} & +50 & 14.35 & 18.61 & 22.90 & 24.20 & $40,232.10$ \\
\hline & +25 & 13.50 & 18.32 & 23.29 & 24.77 & $38,935.20$ \\
\hline & -25 & 12.52 & 18.15 & 24.15 & 25.23 & $29,346.10$ \\
\hline & -50 & 11.37 & 17.86 & 24.96 & 26.45 & $23,806.40$ \\
\hline \multirow{4}{*}{$c_{1}$} & +50 & 12.79 & 18.62 & 23.65 & 25.32 & $52,026.50$ \\
\hline & +25 & 12.79 & 18.62 & 23.65 & 25.32 & $46,040.70$ \\
\hline & -25 & 12.79 & 18.62 & 23.65 & 25.32 & $27,020.30$ \\
\hline & -50 & 12.79 & 18.62 & 23.65 & 25.32 & $19,025.30$ \\
\hline \multirow{4}{*}{$c_{2}$} & +50 & 12.79 & 18.62 & 23.65 & 25.32 & $34,024.30$ \\
\hline & +25 & 12.79 & 18.62 & 23.65 & 25.32 & $34,026.70$ \\
\hline & -25 & 12.79 & 18.62 & 23.65 & 25.32 & $34,104.60$ \\
\hline & -50 & 12.79 & 18.62 & 23.65 & 25.32 & $34,629.10$ \\
\hline \multirow{4}{*}{$c_{3}$} & +50 & 10.56 & 14.75 & 18.67 & 19.29 & $30,215.00$ \\
\hline & +25 & 11.27 & 16.24 & 20.87 & 23.24 & $33,502.00$ \\
\hline & -25 & 15.31 & 21.91 & 25.48 & 28.37 & $44,681.90$ \\
\hline & -50 & 19.76 & 27.14 & 32.89 & 37.38 & $55,714.80$ \\
\hline
\end{tabular}




\section{CONCLUSION}

An order level inventory model for deteriorating items with time-dependent deterioration rate, quadratic demand rate and unit production cost have been present here. The quadratic demand rate is assumed to be time-dependent and the unit production cost is inversely related to the quadratic demand rate and we assumed $\mathrm{a}, \mathrm{b}, \mathrm{c}$ is constant.

This paper is solved by allowing shortages in inventory. Solving highly nonlinear algebraic equations using fourth-order, the average system cost is minimized satisfying the optimization criterion.

\section{Sensitivity Analysis}

Sensitivity analysis is performed by changing (increasing or decreasing) the parameters by $25 \%$ and 50 $\%$ and taking one parameter at a time, keeping the remaining parameters at their original values. From table, the following points are noted

On the basis of the results of table, the following observation can be made.

1. Decrease in the value of the parameter $\alpha$, then $t_{1}{ }^{*}, t_{3}{ }^{*}, t_{4}{ }^{*}$ is increased and $t_{2}{ }^{*}, C^{*}$ is decreased.

2. Decrease in the value of the parameter $\beta$ then $t_{1}{ }^{*}, t_{2}{ }^{*}, t_{3}{ }^{*}, t_{4}{ }^{*}$ and $C^{*}$ is decreased.

3. Decrease in the values of either of the parameters $c_{1}, c_{2}$ then $t_{1}{ }^{*}, t_{2}{ }^{*}, t_{3}{ }^{*}, t_{4}{ }^{*}$ are unchanged but $C^{*}$ value is decreased or increased.

4. Decrease in the value of the parameter $\gamma$ then $t_{1}{ }^{*}, t_{2}{ }^{*}, C^{*}$ is decreased and $t_{3}{ }^{*}, t_{4}{ }^{*}$ is increased.

5. Decrease in the values of either of the parameter $\alpha_{1}$ then $t_{1}{ }^{*}, t_{2}{ }^{*}$ is increased and $t_{3}{ }^{*}, t_{4}{ }^{*}, C^{*}$ is decreased.

6. Decrease in the value of parameter $c_{3}$ then $t_{1}{ }^{*}, t_{2}{ }^{*}, t_{3}{ }^{*}, t_{4}{ }^{*}$ and $C^{*}$ is increased.

\section{REFERENCES}

[1] Bahari-Kashani H. (1989). Replenishment schedule for deteriorating items with time proportional demand. Journal of the Operational Research Society 40, 75-81.

[2] Covert R. P. and Philip G.C. (1973). An EOQ model for items with waybills distribution deterioration. AIIE Transactions 5, 323-326.

[3] Dave U. (1986). An order level inventory model for deteriorating items with variable instantaneous demand and discrete opportunities for replenishment. Opsearch 23, 244-249.

[4] Dave U. and Patel L.K. (1981). ( $\left.T, S_{i}\right)$ Policy inventory model for deteriorating items with time proportional demand. Journal of the Operational Research Society 32, 137-142.

[5] Deb M. and Chaudhuri K.S. (1986). An EOQ model for items with finite rate of production and variable rate of deterioration. Opsearch 23, 175-181.

[6] Donaldson W.A. (1977). Inventory replenishment policy for a linear trend in demand -an analytical solution. Operational Research Quarterly 28, 663-670.

[7] Ghare P.M. and Schrader G.F. (1963). An inventory model for exponentially deteriorating items. Journal of Industrial Engineering 14, 238-243.

[8] Mishra R.B. (1975). Optimum production lot-size model for a system with deteriorating inventory. International Journal of Production Research Society 13, 495-505.

[9] Nahmias S. (1982). Perishable inventory theory-a review. Operational Research 30, 680-708.

[10] Philip G.C. (1974). A generalized EOQ model for items with Weibull distribution deterioration. AIIE Transactions 6, 159-162.

[11] Raafat F. (1991). Survey of literature on continuously deteriorating inventory model. Journal of the Operational Research Society 42, 27-37.

[12] Roychowdhury M. and Chaudhuri K.S. (1983). An order level inventory model for deteriorating items with finite rate of replenishment. Opsearch 20, 99-106.

[13] Shah Y. K. and Jaiswal M.C. (1977). An order level inventory model for a system with constant rate of deterioration. Opsearch 14, 174-184.

[14] Whitin T.M. (1957). Theory of Inventory Management. Princeton University Press, Princeton, NJ. 